\title{
Is It Reasonable to Expect Individuals to Wear Hearing Protectors for Extended Periods?
}

\author{
Warwick Williams \\ National Acoustic Laboratories, Chatswood, NSW, Australia
}

The purpose of this work is to look at the expectations and actions that exist around the use of hearing protectors. Are our expectations for the performance of hearing protectors rational and is the way we expect them to be used reasonable? Perhaps we are expecting too much of hearing protectors and their ability to effectively reduce noise exposure. Better understanding of expectations and requirements along with improvements in technology and design offer the opportunity to markedly improve on the current situation.

hearing protectors comfort discomfort communications

\section{INTRODUCTION}

In the workplace individuals are often expected to wear their personal protective equipment (PPE) for extended periods if not the whole of the work shift which can last from 8 or, nowadays, up to $12 \mathrm{~h}$. Is this a reasonable expectation to place on an individual who may not necessarily perceive that exposure to noise is a particular hazard? PPE that operates as an obvious physical barrier, such as goggles, helmets and steel capped boots, are understood by their presence to offer the elimination or at least significant reduction in the outcome of a serious incident. But where the hazardous exposure is not seen to have any immediate outcome, exposure reduction through the use of PPE is often avoided, delayed or used infrequently. This is often demonstrated in the construction industry where workers are willing to take precautions against immediate physical hazards but are slower and more reluctant to take action against the less immediate threat from noise exposure.

There is no doubt that exposure to excess noise represents a significant hazard to hearing health leading to future hearing loss and there are many readily available documents covering this area $[1,2,3]$. One of the many tools for reducing noise exposure is the use of hearing protectors. There have been many excellent reviews into the effectiveness of hearing protectors the most significant of these being the comparison of laboratory measurements as compared to real in-ear measurements obtained in the field [4]. However, while hearing protectors can be an effective exposure management tool they do have their difficulties and limitations. Recent research suggests that there are three main factors on which hearing protector users comment and which they find difficult [5]: they do not like wearing hearing protectors, hearing protectors are uncomfortable, and they cannot communicate while wearing hearing protectors.

How many of us have actually heard someone say "I like wearing hearing protectors" or words to that effect? In fact does anyone ever clearly say that they like using or wearing any sort of PPE without some sort of caveat? Commonly there will be an implied or inherent understanding that the user may "like" wearing this particular PPE because it is the best of those supplied.

Correspondence and requests for offprints should be sent to Warwick Williams, National Acoustic Laboratories, 126 Greville Street, Chatswood, NSW, 2067, Australia. E-mail: <warwick.williams@nal.gov.au>. 


\section{WEAR-TIME}

The importance of wear-time for hearing protectors has been emphasised by occupational health and safety (OHS) practitioners ever since the difficulty was initially raised by Else [6] and others of not wearing them for the whole of the exposure period. A simple mental calculation demonstrates this point. Let us consider a hypothetically perfect hearing protector that eliminates all hazardous noise to the user. If this protector is worn for half of the exposure time then the total exposure is reduced by half. This reduction is equivalent to a $3-\mathrm{dB}$ change in sound pressure level. Now envisage the case when a worker has a daily exposure level from their combined tasks of $94 \mathrm{~dB}$. Wearing these perfect hearing protectors for half of the exposure time halves the exposure by 3-91 dB. If the permitted daily exposure level $\left(L_{\text {Aeq,8h }}\right)$ is set at $85 \mathrm{~dB}$, e.g., an exposure of $94 \mathrm{~dB}$ is 8 times the permitted exposure level while the exposure reduced to $91 \mathrm{~dB}$ is now 4 times the permitted exposure level. Hence, while the exposure has been significantly reduced, the overall exposure is still 4 times the permitted level.

Figure 1 illustrates the exposure reduction versus total wear-time for several hearing protector attenuations of 30,20 and $10 \mathrm{~dB}$. This figure also clearly emphasises that no matter what the theoretical or actual attenuation of a hearing protector if it is not accepted and worn for the full exposure by the user it will be very ineffective.

When hearing protector users indicate, through questioning, that they use their devices $75 \%$ of the time they are typically only actually effectively receiving in the order of 5-6 dB in overall exposure reduction. So if they are using devices rated at $30 \mathrm{~dB}$ they may be expecting to receive well over $20 \mathrm{~dB}$ if they are not familiar with using nonlinear scales. For comparison, if they wear their safety helmet for $75 \%$ of the time their head is protected for $75 \%$ of the time.

The closeness of the curves presented in Figure 1 also shows that unless the devices are worn for almost the total time of exposure there is really very little difference in the performance between devices. Thus if comfort permits a

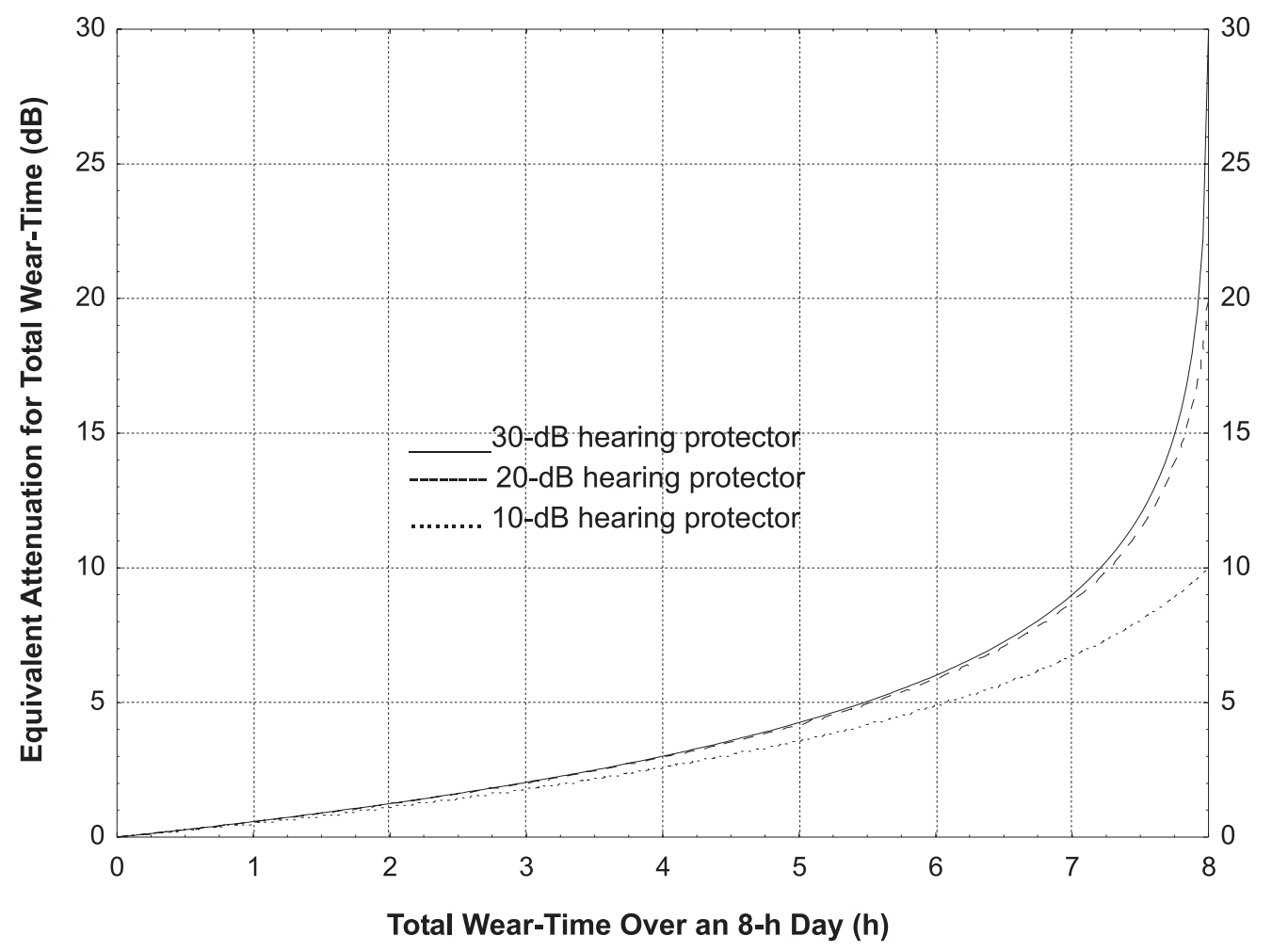

Figure 1. Equivalent exposure attenuation/reduction for total hearing protector wear-time for an 8-h day for a 30-, 20-, and 10-dB protector down the scale [6]. 
device to be worn for a longer time, discomfort becomes a very important factor in noise exposure reduction.

\section{DISCOMFORT}

Discomfort is somewhat more specific than simply not liking to wear hearing protectors but at the same time it is very difficult to define in terms of some form of easily measurable, objective rating system [7]. Discomfort may result from many factors such as the mass of the device, shape of ear cups, the characteristics of the materials that contact the skin, temperature rise inside the ear cup, etc. [8]. It must be remembered that just because an individual cannot put a name to a particular feeling of discomfort that does not mean the feeling of discomfort is not there.

One of the main causes of discomfort often mentioned arises from the clamping force, translated as cushion pressure, holding ear muffs in place. A detailed study of the clamping pressures of a wide range of circum-aural devices revealed that the typical clamping pressures were well over the pressure limits capable of disrupting the blood supply around the ears [9]. This will, at some stage, cause discomfort for most wearers and hence cause for feelings of discomfort and/ or complaint. Figure 2 illustrates the range of pressure and related attenuation.

In general clamping pressures $>1.3 \mathrm{kPa}$ can be expected to cause discomfort through blood flow disruption. The average measured pressure was $2.5 \mathrm{kPa}$ with a range of $\sim 1.6-4.2 \mathrm{kPa}$. Although measurements are yet to be carried out there is no reason to suppose that a similar mechanism could not be operating with pressure along the ear canal from ear plugs due to their lateral expansion characteristics.

If the immediate impact of the offending noise is more uncomfortable than wearing the hearing protector then the effect of the comfort factor is significantly varied in favour of wearing the device [10]. For noise exposure that falls within the range of not being too loud or tolerably loud, more effort will be required through the use of better design and materials to increase the use of hearing protectors. Inconvenience can also

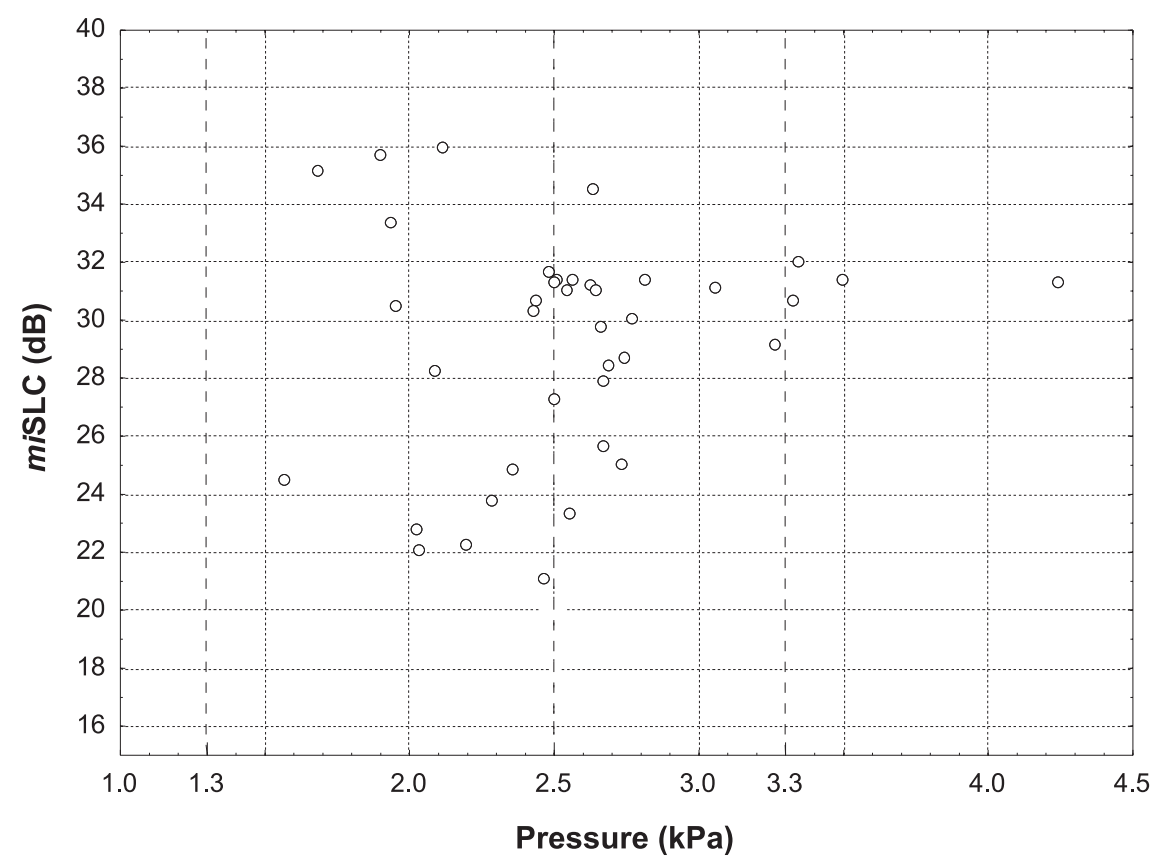

Figure 2. The distribution of attenuation versus clamping pressure for 39 sets of commercially available ear muffs. The mean clamping pressure is indicated by the line at $2.5 \mathrm{kPa}(S D=0.5)$ while the lines at 3.3 and $1.3 \mathrm{kPa}$ show the mean capillary blood pressure at the arterial (inlet) and venous (outlet) sides, respectively [9]. Notes. miSLC—-the mean attenuation a user can expect to get using a particular device. 
be addressed via the supply of information and education perhaps through consideration of the immediate inconvenience of wearing hearing protectors versus the longer term inconvenience of hearing loss.

While there are a few hearing manufacturers who devote considerable effort resources to good design, comfort and quality there are many manufacturers who do not. Unfortunately it is often the case that the buyers and providers of hearing protectors are not the same people who will be expected to wear the devices for $8 \mathrm{~h}$ a day. So comfort is not well considered except for a fitting trial lasting perhaps 1-2 min.

\section{VARIATION IN PERFORMANCE}

Variation in the (acoustic) performance of devices can be a point of concern with the use of hearing protectors. A large study using subjectively gathered test data from over 110 commonly available ear muffs, ear plugs and helmetmounted muffs showed that, in the general case, as the attenuation of a device decreased the variation (standard deviation) in the measured attenuation of the device between test subjects increased significantly [11]. For example, these results show that a hearing protector with a mean attenuation of $30 \mathrm{~dB}$ could be expected to have a standard deviation of $\sim 2 \mathrm{~dB}$ while a protector with a mean attenuation of $20 \mathrm{~dB}$ could be expected to have a standard deviation in the order of $10 \mathrm{~dB}$. Figure 3 demonstrates this point.

An explanation is in order as to why the mean attenuation is used rather than the product labelled attenuation (single number rating, SNR; noise reduction rating, NRR; single level conversion, $\mathrm{SLC}_{80}$, etc.). While it would seem convenient to use the attenuation as labelled on the hearing protector packaging, as required by the local OHS authority, there is a difficulty in that the labelled attenuation through the requirements of the standard includes the standard deviations at the measured octave frequency bands in the attenuation calculation. So mathematically the attenuation as expressed on the labelling is a function of the mean attenuation at each octave band frequency minus its standard deviation. This means that to carry out an appropriate comparison of attenuation versus standard deviation, as presented in the previous paragraph, requires the use of the mean attenuation as experienced by each individual user and the associated standard deviation.

These performance figures imply that the majority of those who use the $30-\mathrm{dB}$ protector experience a range in performance of $\sim 4 \mathrm{~dB}$ while those who use the $20-\mathrm{dB}$ device experience

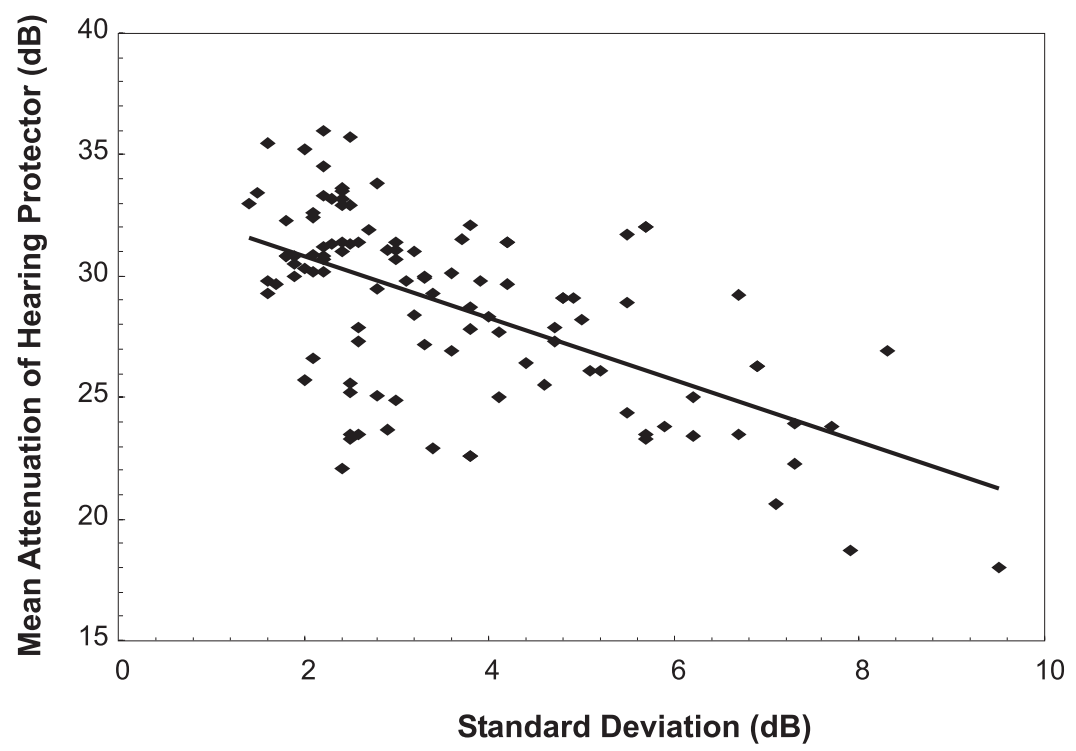

Figure 3. The relationship between mean attenuation and standard deviation for $>100$ hearing protectors with a calculated (straight) line of best fit [11]. 
a range in performance of $\sim 20 \mathrm{~dB}$, with some users experiencing attenuation $>25 \mathrm{~dB}$ and others $<15 \mathrm{~dB}$. Such a wide variation in performance could easily give rise to nonspecific dislike of wearing the protector. In particular this variability of attenuation performance has direct consequences on person-to-person communication (see section 5).

The main difficulty is the fact that the required labelling of hearing protectors includes this variation in performance through the use of the mean attenuation minus one standard deviation for the calculation of SNR and $\mathrm{SLC}_{80}$ while a mean minus two standard deviations is used in the calculation of NRR. Because the standard deviation is used in the calculation of the SNR, e.g., all of this variation in performance is subtly hidden from the end user.

Thus what occurs in practice is that the performance figure of a hearing protector is not reached through intended design but rather through a wide variation in performance. This particularly appears to be the case with lower attenuation devices.

\section{COMMUNICATION}

Users of hearing protectors often expect to be able to still communicate aurally as they do without protectors. Is this a reasonable expectation on the part of the users and those responsible for the workplace? In practice if the noise levels in the area are such that wearing PPE is desirable then the expectation of any sort of normal verbal communication may not be realistic.

Individuals may need to hear warning signals, instructions from supervisors or simply general information from their work environment concerning on-going operations. When regular communication is an expected part of operating in a noisy work environment then planning for communication must be taken into account to avoid miscommunication at all times. Planning for communications can be as involved as supplying appropriate noise excluding communication headsets or as simple as a definitive set of signals as are commonly used for crane operation or ground aircraft controllers.
A survey of the internet will clearly reveal that there are a large number of devices on the market with a wide range of quality and functionality ranging from headsets to personally moulded ear pieces. Anecdotal evidence indicates that the greatest barrier to the use of communication, noise excluding and/or cancelling head-sets in industry is the cost factor. Those responsible are in many cases reluctant to provide appropriate devices because they presumably do not see the value in such added expense.

Commonly most groups who regularly work under noisy conditions will have devised other methods of communicating necessary information through other means such as hand signals, gestures or louder noises. Rarely will communication under such conditions rely on verbal conversation alone, other methods will naturally evolve over time. After all when thick leather gloves are used to handle heavy or rough objects there is no expectation of feel as when using thin, latex surgical gloves.

Expectations of a flat or linear attenuation of all frequencies across the audible spectrum from "musicians"" ear plugs are frequently not met as it is difficult to achieve linear attenuation with mechanical means and for higher levels of noise [12]. Linear attenuation is likely to be satisfactorily addressed in the future through electronic means in conjunction with occlusion. The occluded ear effect, or occlusion, results from blocking the air-borne sound pathway into the ear. This results in a change in the perception of the sound of one's own voice or body generated sounds which can be disturbing to the user [13]. In the case of musicians, the sound of their instrument can vary significantly with an occluded ear. This is more clearly noticeable with the use of ear plugs. It can take some time to get used to this change in sound perception and some users unfortunately never acclimatise and give up using the devices.

\section{FASHION AND PRACTICE}

In spite of the effort of hearing protector manufacturers there seems to be something missing. When it comes to the final analysis 
wearing hearing protectors in whatever form is usually uncomfortable, inconvenient and, most important of all, uncool.

The introduction of hearing protectors that are considered by prospective wearers to be sufficiently "cool" so as to make a fashion, personal or ideological statement will take a significant creative input. This has been to some extent been successfully addressed by industry with respect to safety glasses and UV sun skin protection products, such as creams and lotions. Some manufacturers have started to address the fashion aspect of hearing protectors but so far with very limited success.

The increasing popularity of electronic and active noise cancelling (ANC) headsets of all types offers an opportunity to extend such technology further than has been done to date. The growing popularity of personal stereo players, some including ANC capabilities, has both made wearing headsets more acceptable and assisted the development of the appropriate electronics with corresponding decreasing costs. Manufacturers are currently developing a range of electronic ear muffs and plugs that include ANC with wireless and Bluetooth ${ }^{\circledR}$ communications technology. This coupled with improved passive attenuation design and lighter materials has the potential to result in very user-friendly hearing protectors with an even attenuation across the spectrum.

The design of a perfect hearing protector is not a simple or enviable task. In the meantime more thought needs to be applied to occupational noise management programmes that significantly rely on hearing protector use. A wide range of ideas need to be explored such as hearing protector breaks when individuals may leave the workplace for a period for a quite area where hearing protectors can be safely removed and comfort restored.

\section{CONCLUSION}

By its very nature, exposure to a noise hazard is not well perceived as a threat to personal health and welfare when compared to more immediate threats such as moving machinery. Noise injury is painless and bloodless, and thus ranks much lower on any hazard perception scale. While risk assessment measures will always recognise this fact they also need to emphasise that the noise exposure hazard cannot be ignored. Consequently any noise management programme must fit seamlessly with the management of other workplace hazards.

\section{REFERENCES}

1. Sataloff RT, Sataloff J. Occupational hearing loss. New York, NY, USA: Dekker; 1987.

2. Goelzer B, Hansen CH, Sehrndt GA, editors. Occupational exposure to noise: evaluation, prevention and control. Dortmund, Germany: Federal Institute for Occupational Safety and Health, on behalf of the World Health Organization; 2001. Retrieved February 14, 2009, from: http://www.who.int/occupational_health/ publications/occupnoise/en/index.html

3. Luxon L, Prasher D, editors. Noise and its effects. Chichester, England: Wiley; 2007.

4. Berger EH, Franks JR, Lindgren F. International review of field studies of hearing protector attenuation. In: Axelsson A, Borchgrevink $\mathrm{H}$, Hamernik RP, Hellstrom P, Henderson D, Salvi RJ, editors. Scientific basis of noiseinduced hearing loss. New York, NY, USA: Thieme Medical Publishers; 1996. p. 361-77.

5. Williams W. Barriers to occupational noise management. Acoustique \& Techniques. 2007;49:17- 21 .

6. Else D. A note on the protection afforded by hearing protectors-implications of the equal energy principle, Am Occup Hyg. 1973;16:81-3.

7. Broughton KA. Comfort aspects of ear protection-a review. Bootle, Merseyside, UK: Health and Safety Executive; 1995.

8. Casali JG, Grenell JF. Noise-attenuating earmuff comfort: a brief review and investigation of band-force, cushion and wearing-time effects. Appl Acoust. 1990:29:117-38. 
9. Williams W. Clamping pressure and circum-aural earmuffs. Noise Health. 2007;(9)35:45-50.

10. Hsu YL, Huang CC, Yo CY, Chen CJ, Lien CM. Comfort evaluation of hearing protection. Int J Ind Ergon. 2004;33(6): 543-51.

11. Williams W, Dillon H. Hearing protector performance and standard deviation. Noise Health. 2005;(7)28:51-60.
12. Berger EH. Hearing protection devices. In: Berger EH, Royster LH, Royster JD, Driscoll DP, Layne M, editors. The noise manual. 5th ed. Fairfax, VA, USA: American Industrial Hygiene Association; 2000. p. 379-454.

13. Dillon H. Hearing aids. Stuttgart, Germany: Thieme; 2001. 